Nuclear Security: Countering the Threat From States and Non-State Actors 



\title{
Nuclear Security: Countering the Threat From States and Non-State Actors
}

DAVID BERNELL; BEN WICKIZER; AND MEREDITH BOWERS

\author{
OREGON STATE UNIVERSITY \\ CORVALLIS, OR
}




\section{(ㄷ) (1)}

Nuclear Security: Countering the Threat From States and Non-State Actors by David Bernell, Meredith Bowers E Ben Wickizer is licensed under a Creative Commons Attribution 4.0 International License, except where otherwise noted.

Publication and ongoing maintenance of this textbook is possible due to grant support from Oregon State University Ecampus. Suggest a correction (bit.ly/33cz3Q1)

Privacy (open.oregonstate.education/privacy)

This book was produced with Pressbooks (https://pressbooks.com) and rendered with Prince. 


\section{Contents}

Introduction

Part I

Chapter 1 - The Problem of Nuclear Weapons

Chapter 2 - Nuclear Weapons Treaties and Policies of the United States

Chapter 3 - Global Treaties and International Regimes

Chapter 4 - Nuclear Proliferation: Regional Issues

$\underline{\text { Part II }}$

Chapter 5 - Terrorism and Nuclear Threats

Chapter 6 - Pathways to Nuclear Terror and Key Actors: Assessing the Risk

Chapter 7 - Preventing Nuclear Terrorism

Part III

Chapter 8 - Risks of Nuclear Energy Generation

Chapter 9 - U.S. and Global Safeguards and Policy

Creative Commons License

Recommended Citations

Versioning 



\title{
Introduction
}

\author{
David Bernell
}

The danger posed by nuclear weapons and fissile materials is ever present. The end of the Cold War and the significant reduction in the size of Russian and U.S. nuclear stockpiles did not change this fact of life. There are now nine states that possess nuclear weapons - the United States, Russia, the United Kingdom, France, China, Israel, India, Pakistan, and North Korea - and the number of nuclear weapons in the world in 2019 is estimated to be almost 14,000. In addition, the production of highly-enriched uranium and plutonium continues in several places, while more than 440 civilian nuclear facilities around the world are in operation, posing their own particular risks. When one also considers that non-state actors constitute a significant global danger and the potential for nuclear terrorism, it is clear the need for nuclear security remains paramount. There is no other type of weapon that comes close to doing the level of damage that nuclear weapons can inflict.

At the same time, the use of nuclear technology has an important role in the world. One can make the case the nuclear weapons have helped to keep the peace among the world's major powers since 1945, making large-scale war unthinkable. Nuclear energy provides 10 percent of the world's electricity generation (20 percent in the United States), and newer, safer reactor designs offer the potential for even more. As the world battles the growing dangers of climate change, nuclear energy offers carbon-free, emissions-free electricity to replace fossil fuels. Nuclear technology has also provided significant advances in medicine, while also protecting agricultural production and food supplies. The benefits of nuclear technology have made themselves evident for decades, and for these reasons, the technology is not going away.

It is for this reason that a robust, reliable and effective system must be in place to ensure that the benefits of nuclear materials can be maintained while keeping the dangers at bay. This is what motivates governments, organizations and individuals around the world to control and perhaps eventually eliminate nuclear weapons and dangers they pose, while ensuring the security of fissile materials at civilian nuclear facilities.

This edited volume brings together a number of articles and books written on the subject of nuclear security to provide the reader with a broad and deep understanding of the many issues that surround the subject. It is divided into three parts, covering the challenges associated with nuclear security as it pertains to countries, non-state actors/ terrorism, and civilian nuclear facilities. Each of these areas poses its own unique problems and challenges.

This book is part of an effort at Oregon State University to provide free educational materials to students, as the cost of textbooks has risen significantly and placed a growing financial burden on students. The volume includes books and articles that are open source and available to anyone via the internet, as well as links to copyrighted materials available to students at Oregon State University through the OSU Valley Library. 

PART I

Nuclear Security and State Actors 



\section{Chapter I - The Problem of Nuclear Weapons}

\section{Open Sources (Available to all)}

1. Borrie, J., Caughley, T., \& Wan, W. (2017). Understanding Nuclear Weapon Risks. United Nations Institute for Disarmament Research Report. Chapters 1-6. Retrieved from http://www.unidir.org/files/publications/pdfs/ understanding-nuclear-weapon-risks-en-676.pdf.

2. Roser, M. \& Nagdy, M., (n.d.). Nuclear Weapons. Our World in Data. Retrieved from https://ourworldindata.org/ nuclear-weapons.

\section{Additional Reading (Available to OSU Students through the OSU Valley Library}

3. Reed, T., \& Stillman, D., (2010). Nuclear Express: A Political History of the Bomb and Its Proliferation, Zenith Press. OSU Library E-book, retrieved from https://search.library.oregonstate.edu/permalink/f/ueodtl/ CP71242948080001451.

4. Siracusa, J., (2008). Nuclear Weapons: A Very Short Introduction, Oxford University Press. OSU Library E-book, retrieved from https://search.library.oregonstate.edu/permalink/f/lg9lfhc/

TN_informaworld_s10_1080_09592296_2012_651979. 


\section{Chapter 2 - Nuclear Weapons Treaties and Policies of the United States}

Open Sources (Available to all)

5. Arms Control Association (2018). The new US nuclear strategy is flawed and dangerous. Issue Brief, vol. 10, no. 3 [February 15]. Retrieved from https://www.armscontrol.org/issue-briefs/2018-02/new-us-nuclear-strategyflawed-dangerous-heres-why.

6. Arms Control Association (2019). US-Russian Nuclear Arms Control Agreements, Retrieved from https://www.armscontrol.org/factsheets/USRussiaNuclearAgreements.

7. Colby, Elbridge. The role of nuclear weapons in the US-Russia relationship. Task Force on U.S. Policy Towards Russia, Ukraine, and Eurasia. Carnegie Endowment for International Peace, February 26, 2016. Retrieved from https://carnegieendowment.org/2016/02/26/role-of-nuclear-weapons-in-u.s.-russian-relationship-pub-62901.

8. Council on Foreign Relations (2019). US-Russia nuclear arms control: 1949-2019. Retrieved from https://www.cfr.org/timeline/us-russia-nuclear-arms-control.

9. Gavin, F. J. (2015). Strategies of inhibition: US grand strategy, the nuclear revolution, and nonproliferation. International Security, 40 (1), 9-46. [MIT Open Access Articles]. Retrieved from https://dspace.mit.edu/handle/ $\underline{1721.1 / 100504 .}$

10. Klein, J. J. (2014). Towards a better U.S. nuclear strategy. Journal of Strategic Security, 7 (3), 84-94. Retrieved from https://scholarcommons.usf.edu/jss/vol7/iss3/6/.

11. Nikitin, M.B. The Evolution of Cooperative Threat Reduction: Issues for Congress, CRS Report R43143 [June 13, 2014]. Retrieved from https://fas.org/sgp/crs/nuke/R43143.pdf.

12. Nuclear Threat Initiative (2018), A Roadmap for America's Nuclear Policy and Posture. Retrieved from https://www.nti.org/analysis/reports/statement-nti-ceo-and-co-chair-ernest-j-moniz-and-co-chair-samnunn-us-nuclear-policy-and-posture/.

13. Panda, A. (2018). 'No first use' and nuclear weapons. [July 17]. Council on Foreign Relations. Retrieved from https://www.cfr.org/backgrounder/no-first-use-and-nuclear-weapons.

14. U.S. Department of Defense (2018). Nuclear posture review. Retrieved from https://media.defense.gov/2018/Feb/ 02/2001872886/-1/-1/1/2018-NUCLEAR-POSTURE-REVIEW-FINAL-REPORT.PDF.

15. Woolf, Amy F. (2008). Nuclear weapons in the U.S. national security policy: Past, present, and prospects. CRS Report RL34226 [December 30]. Retrieved from https://fas.org/sgp/crs/nuke/RL34226.pdf.

16. Woolf, Amy F. (2019). Nonstrategic Nuclear Weapons. CRS Report RL32572 [September 6]. Retrieved from https://fas.org/sgp/crs/nuke/RL32572.pdf.

\section{Additional Readings (Available to OSU Students through the OSU Valley Library)}

17. Allison, G., (1969) Conceptual models and the Cuban missile crisis. American Political Science Review, 63 (3). Retrieved from https://www.jstor.org/stable/1954423.

18. Berstein, Barton. (1995). The atomic bombings reconsidered. Foreign Affairs [January/February]. Retrieved from https://www.foreignaffairs.com/articles/asia/1995-01-01/atomic-bombings-reconsidered.

19. Graebner,N. (1990). Ronald Reagan and the Russians. Perspectives on Political Science, 19(2). Retrieved from https://search.library.oregonstate.edu/permalink/f/lg9lfhc/ TN_informaworld_s10_1080_10457097_1990_9944462.

20. Graham, T. (2002). Disarmament Sketches: Three Decades of Arms Control and International Law. University of

6 | Chapter 2 - Nuclear Weapons Treaties and Policies of the United States 
Washington Press. OSU Library E-book, https://search.library.oregonstate.edu/permalink/f/ueodtl/ CP71153966250001451.

21. Gray, C.S., \& Payne, K. (1980) Victory is possible. Foreign Policy [Summer]. Retrieved from https://www.jstor.org/ stable/1148409.

22. Keeny, S., and Panofsky, K. (1981). MAD versus NUTS, Foreign Affairs [Winter]. Retrieved from https://www.jstor.org/stable/20041081.

23. Lugar, R. (2009). Cooperative Threat Reduction and Nuclear Security. Georgetown Journal of International Affairs, 10(1). Retrieved from https://www.jstor.org/stable/43134204.

24. Malewich, B. (2017). Futile superiority: Decision making and the development of new generation nuclear weapons. Comparative Strategy, Vol.36(2), Retrieved from https://search.library.oregonstate.edu/permalink/f/lg9lfhc/ TN_informaworld_s10_1080_01495933_2017_1317537.

25. Schell, J. (2000). The folly of arms control. Foreign Affairs [Sept/Oct]. Retrieved from https://www.foreignaffairs.com/articles/2000-09-01/folly-arms-control.

26. Tannenwald, N. (2018). The vanishing nuclear taboo. Foreign Affairs [November/December]. Retrieved from https://search.library.oregonstate.edu/permalink/f/lg9lfhc/TN proquest2129469004.

17. Allison, G., (1969) Conceptual models and the Cuban missile crisis. American Political Science Review, 63 (3). Retrieved from http://www3.nccu.edu.tw/ lorenzo/Allison\%20Conceptual\%20Models.pdf

21. Gray, C.S., \& Payne, K. (1980) Victory is possible. Foreign Policy [Summer]. Retrieved from https://www.jstor.org/stable/pdf/1148409.pdf 


\section{Chapter 3 - Global Treaties and International Regimes}

\section{Open Sources (Available to all)}

27. Arms Control Association (2019). IAEA Safeguards Agreements. Retrieved from https://www.armscontrol.org/ factsheets/IAEAProtoco.

28. Congressional Research Service (2019). Arms control and nonproliferation: A catalogue of treaties and agreements (CRS Report No. RL33865). Retrieved from https://fas.org/sgp/crs/nuke/RL33865.pdf.

29. Council on Foreign Relations. (2012). The global nuclear nonproliferation regime. Retrieved from https://www.cfr.org/report/global-nuclear-nonproliferation-regime.

30. Nuclear Threat Initiative (2018). Comprehensive nuclear test ban treaty (CTBT). Retrieved from https://www.nti.org/learn/treaties-and-regimes/comprehensive-nuclear-test-ban-treaty-ctbt/.

31. Nuclear Threat Initiative (n.d.). Treaties and Regimes, Nuclear Weapons and Nuclear Safety, Retrieved from https://www.nti.org/learn/treaties-and-regimes/treaties/.

32. Nuclear Threat Initiative (2019). Treaty on the nonproliferation of nuclear weapons (NPT). Retrieved from https://www.nti.org/learn/treaties-and-regimes/treaty-on-the-non-proliferation-of-nuclear-weapons/.

33. Walsh, J. (2005). Learning from Past Successes: The NPT and the Future of Nonproliferation. Paper 41, Weapons of Mass Destruction Commission. Retrieved from https://www.belfercenter.org/publication/learning-past-successnpt-and-future-non-proliferation.

\section{Additional Readings )Available to OSU Students through the OSU Valley Library)}

34. Allison,G. (2010). Nuclear disorder: Surveying atomic threats. Foreign Affairs [January/February]. Retrieved from https://www.jstor.org/stable/i20699776.

35. Sagan, S. (2012). A call for global nuclear disarmament. Nature [July 5], Vol. 487(7405). Retrieved from https://search.library.oregonstate.edu/permalink/f/lg9lfhc/TN gale ofa296572456.

36. Waltz, K. (1981) The Spread of Nuclear Weapons: More May Be Better, The Adelphi Papers, 21:171. Retrieved from https://www.mtholyoke.edu/acad/intrel/waltz1.htm.

37. Weiss, L. (2017). Safeguards and the NPT: Where our current problems began. Bulletin of the Atomic Scientists, 73(5), 328-336. Retrieved from https://search.library.oregonstate.edu/permalink/f/lg9lfhc/ TN informaworld s10 10800096340220171362906.

Additional Readings from above available to students outside of OSU through different links:

34. Allison,G. (2010). Nuclear disorder: Surveying atomic threats. Foreign Affairs [January/February]. Retrieved from https://www.belfercenter.org/publication/nuclear-disorder-surveying-atomic-threats

35. Sagan, S. (2012). A call for global nuclear disarmament. Nature [July 5], Vol. 487(7405). Retrieved from https://fsi-live.s3.us-west-1.amazonaws.com/s3fs-public/Nature-Scott_Sagan.pdf

8 | Chapter 3 - Global Treaties and International Regimes 
Chapter 3 - Global Treaties and International Regimes | 9 


\title{
Chapter 4 - Nuclear Proliferation: Regional Issues
}

\author{
Open Sources (Available to all)
}

38. Atomic Heritage Foundation (2018). Israeli Nuclear Program. Retrieved from https://www.atomicheritage.org/ history/israeli-nuclear-program.

39. Bajoria, J., \& Pan, E. (2010). The U.S.-India nuclear deal. Council on Foreign Relations. Retrieved from https://www.cfr.org/backgrounder/us-india-nuclear-deal.

40. Beehner, L. (2006). Israel's nuclear program and Middle East peace. Council on Foreign Relations. Retrieved from https://www.cfr.org/backgrounder/israels-nuclear-program-and-middle-east-peace.

41. Einhorn, R. (2017). Non-proliferation challenges facing the Trump administration. Arms Control and NonProliferation Series, Paper 15. Brookings Institute. Retrieved from https://www.brookings.edu/research/nonproliferation-challenges-facing-the-trump-administration/.

42. Einhorn, R. (2018). Singapore and beyond: Options for denuclearizing North Korea. Policy Brief. Brookings Institute. Retrieved from https://www.brookings.edu/wp-content/uploads/2018/06/

FP 20180611 singapore and beyond.pdf.

43. Kerr. P. K., \& Nikitin, M. B. (2016). Pakistan's nuclear weapons. CRS Report No. RL34248 [August 1]. Retrieved from https://fas.org/sgp/crs/nuke/RL34248.pdf.

44. Laub, Z. (2019). What is the status of the Iran nuclear agreement? Council on Foreign Relations. Retrieved from https://www.cfr.org/backgrounder/what-status-iran-nuclearagreement?gclid=CjwKCAiAwZTuBRAYEiwAcr67OQppZnd9Mw4bV3Eb7WUqnTK85bFBXyzVotLDesfioa2fCctunDDeBoCINOQAvD_BwE.

45. Parsi, T. (2015). Truce: Iran, the U.S. and the Middle East After the Nuclear Deal. World Politics Review [April 21]. Retrieved from https://www.niacouncil.org/truce-iran-the-u-s-and-the-middle-east-after-the-nuclear-deal/.

46. Pompeo, M. (2018). After the deal: A new Iran strategy. Speech at the Heritage Foundation [May 21]. Retrieved from https://www.heritage.org/defense/event/after-the-deal-new-iranstrategy?utm_source=twitter\&utm_medium=social\&utm_campaign=thf - tw.

47. Roehrig, T. (2013). North Korea's nuclear weapons: Future strategy and doctrine. Belfer Center for Science and International Affiars. Retrieved from https://www.belfercenter.org/publication/north-koreas-nuclear-weaponsfuture-strategy-and-doctrine.

\section{Additional Readings (Available to OSU Students through the OSU Valley Library)}

48. Cohen, A. (2012). The Worst Kept Secret: Israel's Bargain with the Bomb. Columbia University Press. OSU Library Ebook retrieved from https://search.library.oregonstate.edu/permalink/f/ueodtl/CP71134024460001451.

49. Pervez Hoodbhoy, P. \& Mian, Z., (2014). Nuclear fears, hopes and realities in Pakistan. International Affairs, 90 (5). Retrieved from https://search.library.oregonstate.edu/permalink/f/1g9lfhc/TN_wj10.1111/1468-2346.12160.

50. Lang, W.P., \& Johnson, C. (2006). Contemplating the ifs. The National Interest [March 1]. Retrieved from https://nationalinterest.org/article/contemplating-the-ifs-1094

51. Roy, D. (2017). Misunderstanding North Korea. Asia Pacific Issues [August]. Issue 133, 1-8. Retrieved from https://search.library.oregonstate.edu/permalink/f/1g9lfhc/TN_proquest1938122893.

52. Khan, Z. (2013). The US-Indo Civilian Nuclear Deal: The Gainer and the Loser. South Asian Studies, 28(1), $241-257$. Retrieved from https://library.oregonstate.edu/. 
51. Roy, D. (2017). Misunderstanding North Korea. AsiaPacific Issues [August]. Issue 133, 1-8. Retrieved from https://www.eastwestcenter.org/publications/misunderstanding-north-korea

52. Khan, Z. (2013). The US-Indo Civilian Nuclear Deal: The Gainer and the Loser. South Asian Studies, 28(1), 241-257. Retrieved from http://pu.edu.pk/images/journal/csas/PDF/17_V28_1_2013.pdf 

PART II

Nuclear Security and Non-State Actors 



\section{Chapter 5 - Terrorism and Nuclear Threats}

\section{Open Sources (Available to all)}

53. Bunn, M., et al., (2011). US-Russia Joint Threat Assessment on Nuclear Terrorism, Belfer Center for Science and International Affairs, Harvard Kennedy School. Retrieved from https://www.belfercenter.org/publication/usrussia-joint-threat-assessment-nuclear-terrorism.

54. Montgomery, E.B. (2010). Understanding the threat of nuclear terrorism. Center for Strategic and Budgetary Assessments. Retrieved from https://csbaonline.org/research/publications/understanding-the-threat-ofnuclear-terrorism.

55. Mowatt-Larsen, R. (2009). The Armageddon Test. Belfer Center for Science and International Affairs, Harvard Kennedy School. Retrieved from https://www.belfercenter.org/publication/armageddon-test.

56. Sokova, E. K. (2017). Non-state actors and nuclear weapons. In Borrie, J., Caughley, T., \& Wan, W., Understanding nuclear weapon risks (Chapter 8). United Nations Institute for Disarmament Research Report. Retrieved from http://www.unidir.org/files/publications/pdfs/understanding-nuclear-weapon-risks-en-676.pdf.

\section{Additional Readings (Available to OSU Students through the OSU Valley Library)}

57. Barzegar, K. (2014). Nuclear terrorism: An Iranian perspective, Middle East Policy [Spring], 21(1), 29-40. Retrieved from https://search.library.oregonstate.edu/permalink/f/lg9lfhc/TN_wj10.1111/mepo.12055.

58. Zimmerman, P., \& Lewis, J. (2009). The bomb in the backyard. Foreign Policy [Oct. 16]. Retrieved from https://search.library.oregonstate.edu/permalink/f/lg9lfhc/TN_proquest60001293. 


\title{
Chapter 6 - Pathways to Nuclear Terror and Key Actors: Assessing the Risk
}

\author{
Open Sources (Available to all)
}

59. Byman, D. (2015). State sponsor of terror: Iran as global threat. Prepared testimony to House Foreign Affairs Committee Subcommittee on Terrorism, Nonproliferation, and Trade. Retrieved from https://www.brookings.edu/wp-content/uploads/2016/06/11-byman-iran-testimony.pdf.

60. Forest, J. J.F. (2012). Framework for analyzing the future threat of WMD terrorism. Journal of Strategic Security, 5(4), 51-68. Retrieved from https://scholarcommons.usf.edu/jss/vol5/iss4/9/.

61. House, C., (2016). The CBRN Terrorism Threat from the Islamic State, Military Review, Sept/Oct 2016. Retrieved from https://www.armyupress.army.mil/Journals/Military-Review/English-Edition-Archives/SeptemberOctober-2016/.

62. Medalia, J. (2011). "Dirty bombs": Technical background, attack prevention and response, issues for Congress (CRS Report No. R41890) [June 24]. Retrieved from https://fas.org/sgp/crs/nuke/R41890.pdf.

63. MacCalman, M. (2016) A.Q. Khan nuclear smuggling network. Journal of Strategic Security, 9(1), 104-118. Retrieved from https://scholarcommons.usf.edu/jss/vol9/iss1/9/.

64. Nuclear Threat Initiative. (2016). Nuclear Security Index: Building a Framework for Assurance, Accountability, and Action (3rded). Retrieved from http://www.ntiindex.org/wp-content/uploads/2016/03/NTI_2016-IndexReport_MAR-25-2.pdf.

65. Sokolski, H. (2014). Nuclear weapons gone missing: What does history teach? The Strategic Studies Institute Publications Office, United States Army War College Press. Retrieved from https://ssi.armywarcollege.edu/ nuclear-weapons-materials-gone-missing-what-does-history-teach/

\section{Additional Readings (Available to OSU Students through the OSU Valley Library)}

66. Allison, G., \& Tobey, W. H. (2016). Could there be a terrorist Fukushima. New York Times (April 4). Retrieved from https://www.nytimes.com/2016/04/05/opinion/could-there-be-a-terrorist-fukushima.html.

67. Kuperman, A. J. (2013). Nuclear terrorism and global security: The challenge of phasing out highly enriched uranium. New York, NY: Routledge. OSU Library E-book, https://search.library.oregonstate.edu/permalink/f/ ueodtl/CP71310148450001451.

68. Lawlor, B. (2011). The Black Sea: Center of the nuclear black market. Bulletin of the Atomic Scientists, 2011. Retrieved from https://search.library.oregonstate.edu/permalink/f/lg9lfhc/ TN_informaworld_s10_1177_0096340211426506.

69. Lieber K., \& Press, D. (2013). "Why States Won't Give Nuclear Weapons to Terrorists," International Security [June]. Retrieved from https://search.library.oregonstate.edu/permalink/f/lg9lfhc/TN_mitpress10.1162/ISEC_a_00127.

70. Potter, W. C., Ferguson, C. D., \& Spect, L. S. (2004). The four faces of nuclear terror and the need for a prioritized response. Foreign Affairs [May/June]. Retrieved from https://search.library.oregonstate.edu/permalink/f/ 1g9lfhc/TN_proquest214296764.

71. Rezaei, F. (2016). Shopping for Armageddon: Islamist groups and nuclear terror. Middle East Policy, 23(3), 112-132. Retrieved from https://search.library.oregonstate.edu/permalink/f/lg9lfhc/TN_wj10.1111/mepo.12221. 
64. Allison, G., \& Tobey, W. H. (2016). Could there be a terrorist Fukushima. New York Times (April 4). Retrieved from https://www.nytimes.com/2016/04/05/opinion/could-there-be-a-terrorist-fukushima.html.

69. Lieber K., \& Press, D. (2013). Why States Won't Give Nuclear Weapons to Terrorists," International Security [June]. Retrieved from https://www.belfercenter.org/publication/why-states-wont-give-nuclear-weaponsterrorists

71. Rezaei, F. (2016). Shopping for Armageddon: Islamist groups and nuclear terror. Middle East Policy, 23(3), 112-132. Retrieved from https://www.mepc.org/journal/shopping-armageddon-islamist-groups-and-nuclearterror 


\title{
Chapter 7 - Preventing Nuclear Terrorism
}

\author{
Open Sources (Available to all)
}

72. Bunn, M., Malin, M. B., Roth, N., \& Tobey, W. H. (2016) Preventing nuclear terrorism: Continuous improvement or dangerous decline? Belfer Center for Science and International Affairs, Harvard Kennedy School. Retrieved from https://www.belfercenter.org/publication/preventing-nuclear-terrorism-continuous-improvement-ordangerous-decline.

73. Levi, M. A. (2008). Deterring state sponsorship of nuclear terrorism. Council Special Report No. 39. Council on Foreign Relations. Retrieved from https://www.cfr.org/content/publications/attachments/ Nuclear_Deterrence_CSR39.pdf.

74. Klein, J. J. (2012). Deterring and dissuading nuclear terrorism. Journal ofStrategic Security, 5(1), 15-30. Retrieved from https://scholarcommons.usf.edu/jss/vol5/iss1/6/.

75. United Nations. (2005). U.N. International Convention for the Suppression of Acts of Nuclear Terrorism. Retrieved from https://treaties.un.org/doc/db/terrorism/english-18-15.pdf.

76. U.S. Department of State. (2015). The global challenge of chemical, biological, radiological or nuclear (CBRN) terrorism. Country Reports on Terrorism 2015 [Chapter 4]. Retrieved from https://2009-2017.state.gov/j/ct/rls/ crt/2015//index.htm.

\section{Additional Readings (Available to OSU Students through the OSU Valley Library)}

77. Allison, G. (2004). How to stop nuclear terror. Foreign Affairs [Jan/Feb]. Retrieved from https://search.library.oregonstate.edu/permalink/f/lg9lfhc/TN_proquest214293059.

78. Levi, M. (2008). Stopping nuclear terrorism. Foreign Affairs [Jan/Feb]. Retrieved from https://search.library.oregonstate.edu/permalink/f/lg9lfhc/TN_jstor_archive_620020273.

79. Korbatov, A.B., Suzuki, E., \&Goldblum, B. (2015). The fight against nuclear terrorism needs global cooperation, Bulletin of the Atomic Scientists, 71(5). Retrieved from https://search.library.oregonstate.edu/permalink/f/ 1g9lfhc/TN_informaworld_s10_1177_0096340215590795.

80. Mueller, J. (2009). Think again: Nuclear weapons. Foreign Policy [Dec 18]. Retrieved from from https://search.library.oregonstate.edu/permalink/f/lg9lfhc/TN_gale_ofa216896318.

81. Stone, J. (2009). Al-Qaeda, Deterrence and Weapons of Mass Destruction, Studies in Conflict and Terrorism, 32(9), 763-775. Retrieved from https://search.library.oregonstate.edu/permalink/f/lg9lfhc/ TN_informaworld_s10_1080_10576100903109693. 


\section{PART III}

Nuclear Security and Civilian Facilities 



\section{Chapter 8 - Risks of Nuclear Energy Generation}

\section{Open Sources (Available to all)}

82. Baylon, C., Brunt, R., and Livingstone, D. (2015). Cybersecurity at civil nuclear facilities: Understanding the risks, Chatham House Report. Retrieved from https://www.chathamhouse.org/publication/cyber-security-civilnuclear-facilities-understanding-risks.

83. Crenshaw, M. (2017). Deterring nuclear terrorism: Insider threats to nuclear facilities. NAPSNet Special Reports. Retrieved from https://nautilus.org/napsnet/napsnet-special-reports/deterring-nuclear-terrorism-insiderthreats-to-nuclear-facilities/.

84. ElBaradei, M. (2008). Nuclear energy: The need for a new framework. Retrieved from https://www.iaea.org $/$ newscenter/statements/nuclear-energy-need-new-framework.

85. Feiveson, H, Glaser, A, Miller, M., \& Scheinman, L. (2008). Can future nuclear power be made proliferation resistant? Center for International and Security Studies at University of Maryland. Retrieved from http://cissmdev.devcloud.acquia-sites.com/sites/default/files/papers/future_nuclear_power.pdf. (inactive link as of $5 / 28 / 2021)$

86. Lahidji, R. (2017). The safety of nuclear weapon and materials: Lessons from the assessment of nuclear power plant risks. In Borrie, J., Caughley, T., \& Wan, W., Understanding nuclear weapon risks(Chapter 7). United Nations Institute for Disarmament Research Report. Retrieved from http://www.unidir.org/files/publications/pdfs/ understanding-nuclear-weapon-risks-en-676.pdf.

87. Lamb, R. \& Peteru, A. (2019). Fissile Materials Security in Civilian Facilities: A System Story, Foundation for Inclusion. Retrieved from the Center for International Security Studies at Maryland, University of Maryland, https://cissm.umd.edu/research-impact/publications/fissile-materials-security-civilian-facilities-system-story.

88. Union of Concerned Scientists. (2016). Nuclear plant security. (Feb. 25). Retrieved from https://www.ucsusa.org/ nuclear-power/nuclear-plant-security\#.W0pyBi3Myu4.

\section{Additional Readings (Available to OSU Students through the OSU Valley Library)}

89. Bjornard, T., and Morgan, J. (2012) Integrating safeguards and security into nuclear facility design, Nuclear Technology, 179(1). Retrieved from https://search.library.oregonstate.edu/permalink/f/lg9lfhc/ TN informaworld s10 13182 NT12 A14075.

90. Fuhrmann, M. (2009). Spreading temptation: Proliferation and peaceful nuclear cooperation agreements, International Security, 34(1). Retrieved from https://search.library.oregonstate.edu/permalink/f/lg9lfhc/ TN_mitpress10.1162/isec.2009.34.1.7.

Additional Readings from above available to students outside of OSU through different links:

89. Bjornard, T., and Morgan, J. (2012) Integrating safeguards and security into nuclear facility design, Nuclear Technology, 179(1). Retrieved from https://www.tandfonline.com/doi/pdf/10.13182/ $\underline{\text { NT12-A14075?needAccess }=\text { true }}$ 
90. Fuhrmann, M. (2009). Spreading temptation: Proliferation and peaceful nuclear cooperation agreements, International Security, 34(1). Retrieved from https://www.belfercenter.org/sites/default/files/legacy/files/ Spreading-Temptation-Proliferation-and-Peaceful-Nuclear-Cooperation-Agreements.pdf 


\section{Chapter 9 - U.S. and Global Safeguards and Policy}

\section{Open Sources (Available to all)}

91. Holt, M., \& Andrews, A. (2014). Nuclear power plant security and vulnerabilities. CRS Report No. RL34331 [January 3]. Retrieved from https://fas.org/sgp/crs/homesec/RL34331.pdf.

92. International Atomic Energy Agency. (2011). Nuclear Security Recommendations on Physical Protection of Nuclear Material and Nuclear Facilities (INFCIRC/225/Revision 5). Retrieved from https://www.iaea.org/publications/ 8629/nuclear-security-recommendations-on-physical-protection-of-nuclear-material-and-nuclear-facilitiesinfcirc/225/revision-5.

93. International Atomic Energy Agency. (2008). Nuclear security culture. IAEA Nuclear Security Series No. 7: Implementing Guide. Retrieved from https://www-pub.iaea.org/MTCD/Publications/PDF/Pub1347_web.pdf.

94. U.S. Nuclear Regulatory Commission. (2015). Protecting our nation: A Report of the U.S. Nuclear Regulatory Commission. Office of Nuclear Security and Incident Response. Retrieved from https://www.nrc.gov/docs/ ML1523/ML15232A263.pdf.

95. Van Dine, A., Assante, M., \& Soutland, P. (2016). Outpacing cyber threats: Priorities for cyber security at nuclear facilities. Nuclear Threat Initiative. Retrieved from http://www.nti.org/media/documents/ NTI_CyberThreats__FINAL.pdf.

\section{Additional Readings (Available to OSU Students through the OSU Valley Library)}

96. Deutch, J., Kanter, A., Moniz, E., \& Poneman, D. (2004). Making the world safe for nuclear energy. Survival, 46(4), 65-79. Retrieved from https://search.library.oregonstate.edu/permalink/f/lg9lfhc/ TN_informaworld_s10_1080_00396330412331342466.

97. Holdren, J. P. (1983). Nuclear power and nuclear weapons: the connection is dangerous. Bulletin of the Atomic Scientists, 39(1), 40-45. Retrieved from https://search.library.oregonstate.edu/permalink/f/lgglfhc/ TN_gale_ofa2572563.

98. Rockwood, L., (2018). How the IAEA verifies if a country's nuclear program is peaceful or not: The legal basis. Bulletin of the Atomic Scientists, 74(5), 317-325. Retrieved from https://search.library.oregonstate.edu/permalink/ f/lg9lfhc/TN_wos000443845800004.

99. Frank R. Spellman, F. R., \& Stoudt, M. L. (2011). Nuclear infrastructure protection and homeland security. Lanham, MD: Government Institutes. OSU Library E-book, retrieved from https://search.library.oregonstate.edu/ permalink/f/ueodtl/CP71189509150001451.

100. Spinrad, B. (1983). Nuclear power and nuclear weapons: the connection is tenuous. Bulletin of the Atomic Scientists, 39(2), 42-47. Retrieved from https://search.library.oregonstate.edu/permalink/f/lg9lfhc/ TN informaworld s10 $1080 \quad 00963402 \quad 1983 \quad 11458951$. 



\section{Creative Commons License}

This work is licensed by David Bernell, Meredith Bowers \& Ben Wickizer (C 2019) under a Creative Commons Attribution 4.0 International License (CC BY)

You are free to:

Share - copy and redistribute the material in any medium or format

Adapt - remix, transform, and build upon the material

The licensor cannot revoke these freedoms as long as you follow the license terms.

Under the following terms:

Attribution - You must give appropriate credit, provide a link to the license, and indicate if changes were made. You may do so in any reasonable manner, but not in any way that suggests the licensor endorses you or your use.

No additional restrictions - You may not apply legal terms or technological measures that legally restrict others from doing anything the license permits. 


\section{Recommended Citations}

APA outline:

Source from website:

- (Full last name, first initial of first name). (Date of publication). Title of source. Retrieved from https://www.someaddress.com/full/url/

Source from print:

- (Full last name, first initial of first name). (Date of publication). Title of source. Title of container (larger whole that the source is in, i.e. a chapter in a book), volume number, page numbers.

Examples

If retrieving from a webpage:

- Berndt, T. J. (2002). Friendship quality and social development. Retrieved from insert link.

If retrieving from a book:

- Berndt, T. J. (2002). Friendship quality and social development. Current Directions in Psychological Science, 11, 7-10.

MLA outline:

Author (last, first name). Title of source. Title of container (larger whole that the source is in, i.e. a chapter in a book), Other contributors, Version, Number, Publisher, Publication Date, Location (page numbers).

Examples

- Bagchi, Alaknanda. "Conflicting Nationalisms: The Voice of the Subaltern in Mahasweta Devi's Bashai Tudu." Tulsa Studies in Women's Literature, vol. 15, no. 1, 1996, pp. 41-50.

- $\quad$ Said, Edward W. Culture and Imperialism. Knopf, 1994.

Chicago outline:

Source from website:

- Lastname, Firstname. "Title of Web Page." Name of Website. Publishing organization, publication or revision date if available. Access date if no other date is available. URL .

26 | Recommended Citations 
Source from print:

- Last name, First name. Title of Book. Place of publication: Publisher, Year of publication.

Examples

- $\quad$ Davidson, Donald, Essays on Actions and Events. Oxford: Clarendon, 2001. https://bibliotecamathom.files.wordpress.com/2012/10/essays-on-actions-and-events.pdf.

- $\quad$ Kerouac, Jack. The Dharma Bums. New York: Viking Press, 1958. 


\section{Versioning}

This page provides a record of changes made to this publication. Each set of edits is acknowledged with a 0.01 increase in the version number. The exported files, available on the homepage, reflect the most recent version.

If you find an error in this text, please fill out the form at bit.ly/33cz3Q1

\begin{tabular}{|l|l|l|l|}
\hline Version & Date & Change Made & Location in text \\
\hline 0.1 & MM/DD/YYYY & & \\
\hline 0.11 & $10 / 08 / 2020$ & Links to external sources updated & All \\
\hline & & & \\
\hline
\end{tabular}

